Chapman University

Chapman University Digital Commons

Pharmacy Faculty Articles and Research

School of Pharmacy

9-22-2010

\title{
Morphological Study of Emulsion-Assisted Cholesterol Precipitation Processes
}

Vuk Uskoković

Chapman University, uskokovi@chapman.edu

Follow this and additional works at: http://digitalcommons.chapman.edu/pharmacy_articles

Part of the Cardiovascular Diseases Commons, and the Medical Biochemistry Commons

\section{Recommended Citation}

Uskoković V. Morphological study of emulsion-assisted cholesterol precipitation processes. Molecular Crystals and Liquid Crystals. 2007;474:77-88. doi: 10.1080/15421400701693567

This Article is brought to you for free and open access by the School of Pharmacy at Chapman University Digital Commons. It has been accepted for inclusion in Pharmacy Faculty Articles and Research by an authorized administrator of Chapman University Digital Commons. For more information, please contact laughtin@chapman.edu. 


\section{Morphological Study of Emulsion-Assisted Cholesterol Precipitation Processes}

\section{Comments}

This is an Accepted Manuscript of an article published in Molecular Crystals and Liquid Crystals, volume 474, in 2007, available online: http:/ / www.tandfonline.com/doi/abs/10.1080/15421400701693567.

\section{Copyright}

Taylor \& Francis 


\title{
Morphological Study of Emulsion-Assisted Cholesterol Precipitation Processes
}

\author{
Vuk Uskoković \\ Center for Advanced Materials Processing, Clarkson University, Potsdam, NY, USA
}

\begin{abstract}
Crystallization of cholesterol in chemical conditions that involve the presence of emulsions of three different types of surface active agents - cationic, anionic and nonionic - was investigated by means of scanning and transmission electron microscopic analyses. In contrast with the previous attempts to modify the typical plate- and needleshaped character of biaxially grown cholesterol crystals, the present study indicates that spherical "templates" of fine emulsion droplets may impose spherical morphologies to cholesterol particles nucleated within.
\end{abstract}

\section{Introduction}

Cholesterol presents one of the essential biochemical compounds in the animal world, involved in numerous body-functioning tasks, ranging from the synthesis of bile acids and steriod hormones in vivo to maintaining a proper transport across cellular membranes ${ }^{1}$. On the other hand, due to disbalanced relationships between cholesterol intake and deposition rate with the rate of its solubilization through the action of micellar, vesicular and bilayer cleansing agents in bile and specific lipoprotein complexes in blood, cholesterol may form excessive deposits on the flow-paths of some body fluids and cause problematic health issues that range from gallstone formation to intestinal lumen deposits to atherosclerotic plaque. Thereupon, due to potential development of capability towards reversible control, investigation of cholesterol crystallization into novel morphologies presents a task with large significance for both the areas of biomedicine and chemotherapeutics and the fundamental understanding of complex biochemistry of life.

All of the previous attempts to obtain any morphology of cholesterol crystalline deposits other than the ones composed of typical plate-shaped and needle-shaped crystals by means of mere chemical manipulation did not succeed. Despite the fact that the effects of solvent type $\mathrm{e}^{2,3,4}$, non-solvent phases ${ }^{5}$, temperature ${ }^{2}, \mathrm{pH}^{6}$, electrolytes ${ }^{7}$, the dynamics of solvent systems ${ }^{8}$, magnetic field ${ }^{9}$, mineral substrates ${ }^{10}$ and co-existing phases ${ }^{11,12}$ (such as hydroxyapatite deposits, often found interspersed within cholesterol layers in atherosclerotic plaques ${ }^{13,14}$ ), model bile composition ${ }^{15,16,17}$, various medicinal plants ${ }^{18}$ and synthetic biochemical compounds (including phospholipids ${ }^{19}$, cholic acid ${ }^{20}$ and other sterols $^{21}$ ) on the process of crystallization and dissolution of cholesterol were previously evidenced, the inherent structural tendency of cholesterol molecules to adopt biaxiallygrown formations in solid state defied all the imposed environmental constraints. The observed variations in surface polarity of cholesterol crystals with the nature of the solvent $^{22}$ and the multifunctional biological role of cholesterol, that includes cell membrane flexibility mediation and transmembrane signal messaging ${ }^{1}$, may be indicators of this intrinsic structural flexibility. The reason for the ostensibly inevitable formation of biaxially-grown crystals can be found in the disparity between interlayer and intralayer 
interactions between cholesterol molecules in solid state. Namely, comparatively stronger, hydrogen bonds predominantly figure as intermolecular links within the individual layers of cholesterol molecules, whereby weaker, van der Waals' bonds are predominantly involved in stacking the layers along the c axis. A faster crystal growth in the bilayer plane comparing to the growth in the direction of the c axis is, therefore, energetically more favorable within these conditions. However, as is going to be shown throughout the following text, due to the existence of micellar effects that - via limiting and templating nuclei and particle growth - decrease, globulize and uniformize the resulting crystal forms, emulsion-assisted crystallization can be regarded as an effective means to decrease the dimensions of cholesterol plate-shaped crystals to almost nanosized level and obtain well-dispersed cholesterol crystals with spherical morphologies.

\section{Experimental part}

\subsection{Materials}

The chemicals used in the presented procedures of synthesis were: cholesterol (99+\%, Alfa Aesar), Triton X-100 (Rohm and Haas), cetyltrimethylammonium bromide CTAB (99 \%, Alfa Aesar), sodium n-dodecyl sulfate - SDS (99+\%, Alfa Aesar), and 1hexanol (J. T. Baker).

\subsection{Methods of synthesis}

In the first presented method, $1 \mathrm{ml}$ of Triton X-100, $10 \mathrm{ml}$ of 1-hexanol and 2.2 $\mathrm{ml}$ of distilled water were successively poured into a beaker, resulting in the formation of a white turbid mixture. Twenty milligrams of commercial cholesterol were introduced in such prepared mixture, followed by the addition of $32 \mathrm{ml}$ of 1-hexanol. The mixture was then stirred for 30 minutes by means of a magnetic bar, until the liquid became completely transparent. Twelve milliliters of distilled water were then rapidly poured into the emulsion, resulting in the formation of opaque white dispersion. Samples of the suspension were after 10 min of agitated aging taken through pipette, poured on top of the sample carriers for SEM and TEM analyses, and let dry in vacuum.

Within the second method, $36 \mathrm{mg}$ of SDS were dissolved in $10 \mathrm{ml}$ of 1-hexanol and $2 \mathrm{ml}$ of distilled water. Twenty milligrams of commercial cholesterol were introduced in such obtained emulsion, with the later addition of $42 \mathrm{ml}$ of 1-hexanol and 2 $\mathrm{ml}$ of water. In the ceaselessly agitated emulsion, which might be otherwise settling into thoroughly transparent biphase mixture, $12 \mathrm{ml}$ of distilled water was abruptly introduced. The emulsion was further stirred for 5 minutes, let remain still for 10 minutes, with a dispersion sample poured on top of an SEM sample carrier afterwards, dried in air and viewed under the SEM.

In the third method, $33 \mathrm{mg}$ of CTAB were dissolved in $10 \mathrm{ml}$ of 1-hexanol and 2 $\mathrm{ml}$ of distilled water. Twenty milligrams of commercial cholesterol were introduced in such obtained emulsion. Into this transparent, but visibly emulsified liquid, additional 5 $\mathrm{ml}$ of 1-hexanol were introduced. In so prepared, magnetically agitated emulsion, $5 \mathrm{ml}$ of distilled water were then abruptly added, resulting in the formation of white dispersion of 
cholesterol particles. After 10 minutes of agitated aging, a dispersion sample was poured on top of an SEM sample carrier, dried and observed under the SEM.

In the fourth method, $1 \mathrm{~g}$ of CTAB was dissolved in $18.5 \mathrm{ml}$ of 1 -hexanol and 0.5 $\mathrm{ml}$ of distilled water. In such prepared emulsion, $330 \mathrm{mg}$ of cholesterol was introduced, followed by the addition of $3 \mathrm{ml}$ of water, and resulting in the formation of white, emulsified dispersion. Magnetic stirring was subsequently discontinued and the emulsion remained unstirred for the following $24 \mathrm{~h}$, when the sample for SEM analysis was taken.

\subsection{Characterization}

Morphologies of the prepared cholesterol particles were examined with using scanning electron microscopy (SEM) and field-emission scanning electron microscopy (FE-SEM), whereby the process of melting of submicron-sized cholesterol particles was observed by means of transmission electron microscopy (TEM).

\section{Results and discussion}

In the previous study ${ }^{23}$, the isoelectric point of cholesterol crystals was determined as existing in relatively low acidic $\mathrm{pH}$ range. This observation is consistent with the previous results reported in the literature ${ }^{24}$, according to which the isoelectric point of cholesterol particles might have been deduced as present at $\mathrm{pH}=2.7$. Negative surface charge of cholesterol particles at neutral $\mathrm{pH}$ values is a consequence of the constitutive hydroxyl groups that typically come to protrude crystal surfaces when the crystallization is performed in polar solvents. Microemulsions and emulsions based on octylphenol ethoxylate (Triton X-100), sodium n-dodecyl sulfate (SDS) and cetyltrimethylammonium bromide (CTAB), as nonionic, anionic and cationic surfactants, respectively, were chosen as emulsified media for the study of emulsion-assisted precipitation of cholesterol particles. Triton X-100 as a biodegradable nonionic surfactant has previously been known as an excellent dispersant for oil-in-water emulsions. On the other hand, the modification of physical effects that govern the phase transition interaction of negatively charged cholesterol particles at neutral $\mathrm{pH}$ conditions was investigated by the introduction of electrically charged molecular moieties of CTAB and SDS within cholesterol precipitation processes.

After three-minute aging of the precipitate, preparation of which is described in section 2.2, involving the use of Triton X-100-based emulsion, cholesterol crystallizes in form of square-shaped particles with $0.1-0.5 \mu \mathrm{m}$ in size, as can be evidenced from SEM images in Fig.1. In addition to square-shaped particles, bilayered-substructured micelles and other complex self-assembly patterns that incorporate the former solids were observed by means of FE-SEM analysis (Fig.2). As a regular constituent of cellular membranes and an amphiphilic compound, cholesterol has a tendency for positioning at oil-water interface (which was evidenced by using centrifugation treatment and consequently inducing segregation of aqueous and alcoholic phases with a thin white layer of dispersed cholesterol particles in-between, although easily re-dispersed in reemulsified mixture, obtained again by only mild shaking), which might present the background of origins of the observed complex self-assembly patterns. The cholesterol particles, however, melt and undergo shape and phase modification under the influence of 
high-voltage accelerated TEM electron beam, as can be seen from TEM images in Figs.35. Nanosized melted cholesterol spheres have $50-100 \mathrm{~nm}$ in diameter, whereby an increased density of the constitutive matter towards edges of the particles comparing to the particle cores is consistent with the previous observation of similarly structured melted cholesterol droplets ${ }^{25}$. As the heat content of cholesterol deposits increases, sharpedged crystallites become rounded and in vicinity of the melting point form spherical aggregates $^{25}$.

Significant charging effects evidenced during the SEM analyses of cholesterol samples at regular acceleration voltages $(\sim 15 \mathrm{kV})$, and frequently observed bending, yielding and ripping of cholesterol platelet-shaped crystalline structures, presented an immediate indication of significant susceptibility of cholesterol crystalline deposits upon the influence of electron beams. Thermal absorption of electrons during TEM analyses, therefore, presents the reason for the formation of melted cholesterol droplets, typically composed of distinct spherical membranes that correspond to boundary outlines of cholesterol droplets. Debuigne et al. have been so far the only research team that has reported a microemulsion-assisted cholesterol preparation route ${ }^{26}$, as well as the corresponding formation of nanosized spherical cholesterol particles with $3-7 \mathrm{~nm}$ in size. However, taking into account that: a) TEM was in this case also used to characterize the morphology of re-crystallized cholesterol particles and calculate the average particle size thereof; b) individual cholesterol molecule posesses $~ 1.7 \mathrm{~nm}$ in length, leads to the conclusion that the observed were probably only the remnants of melted or even decomposed initial cholesterol crystals under the influence of sufficiently high local temperatures produced by TEM electron beam under standard measurement conditions. More of the interesting features of the process of melting of typical, plate- or needleshaped cholesterol crystals into individual cholesterol spheres may be evidenced by employing TEM analysis, and are presented in the following images. Cholesterol crystals preserving their sharp edges, albeit the decomposition of their inner layers into spherical particles during the TEM imaging, are presented in Fig.4a, whereby Fig.4b shows a single, less than $10 \mathrm{~nm}$ sized cholesterol particle transforming into melted spheres under the influence of TEM observation. The process of transformation of a needle-shaped cholesterol crystal into spine-like array of spherical particles is presented in Fig.5.

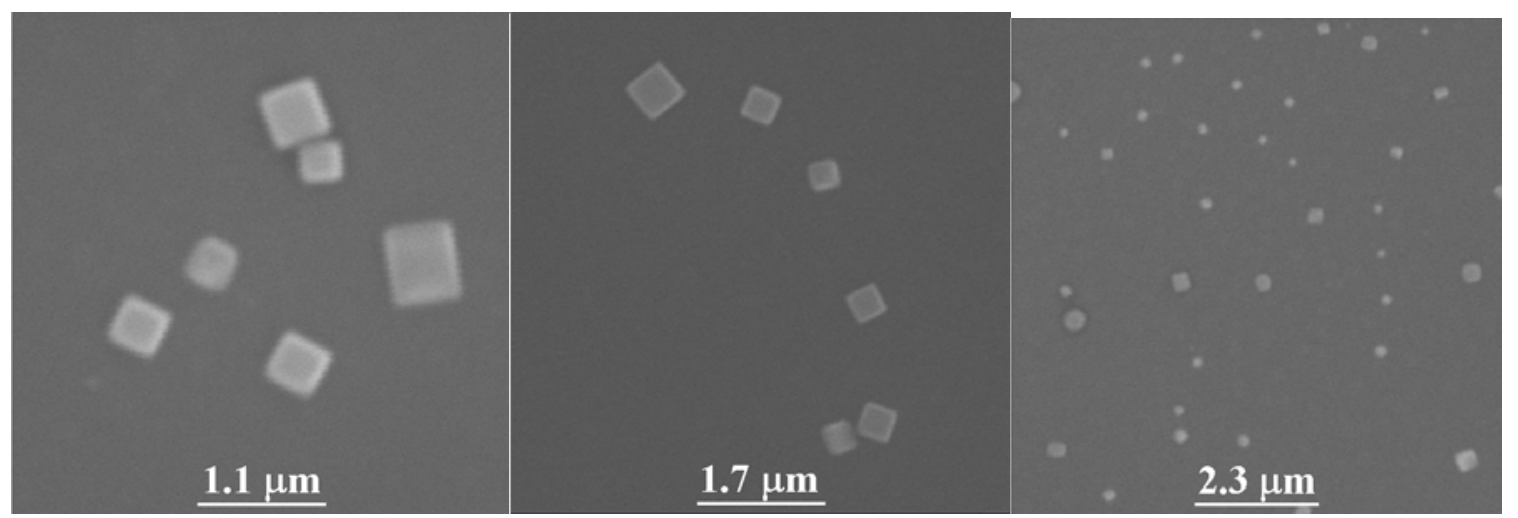

Fig.1. Sub-micron sized, square-shaped cholesterol particles obtained by precipitation in Triton X-100/1hexanol/water microemulsion. 


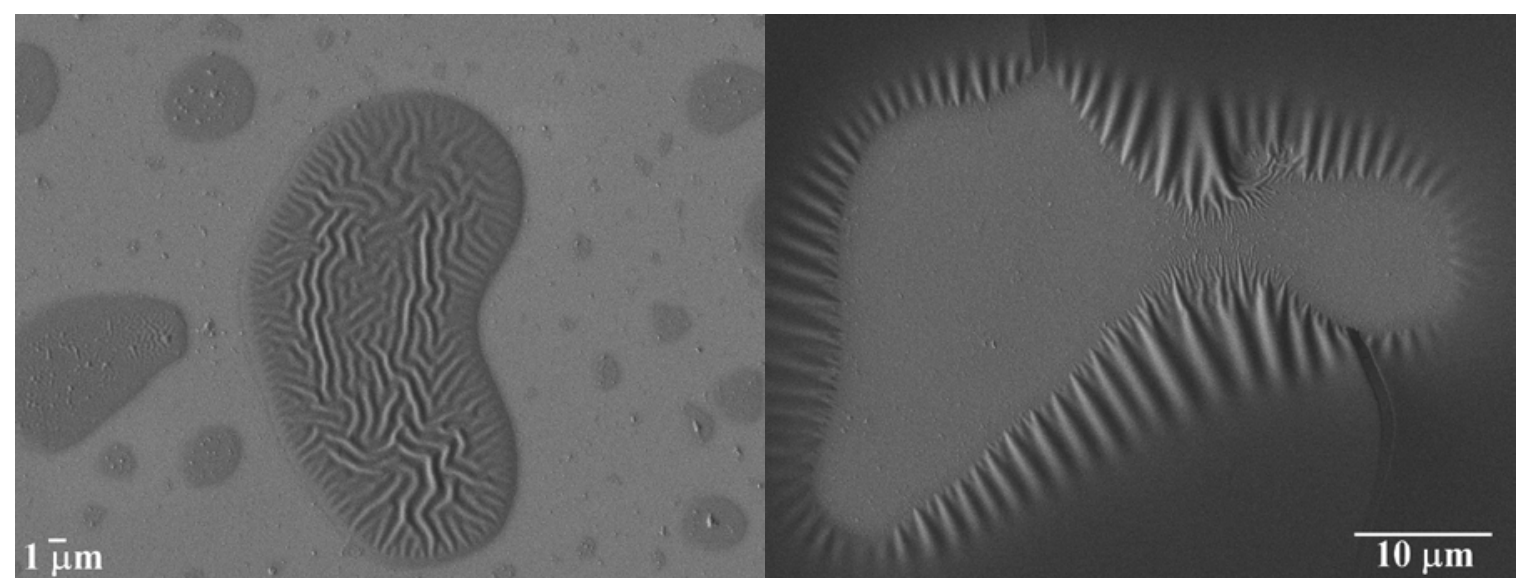

Fig.2. FE-SEM images of the self-assembled patterns in the emulsion system comprising Triton X-100, cholesterol, 1-hexanol and water.

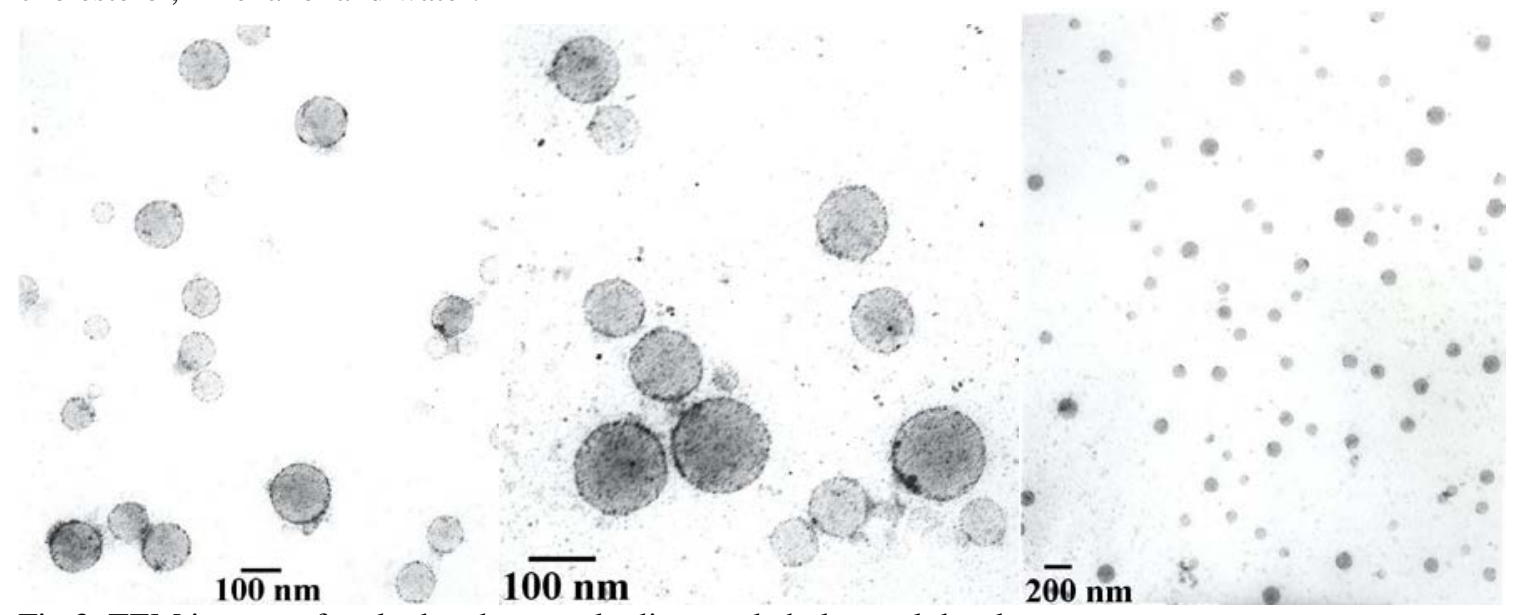

Fig.3. TEM images of melted and narrowly dispersed cholesterol droplets.
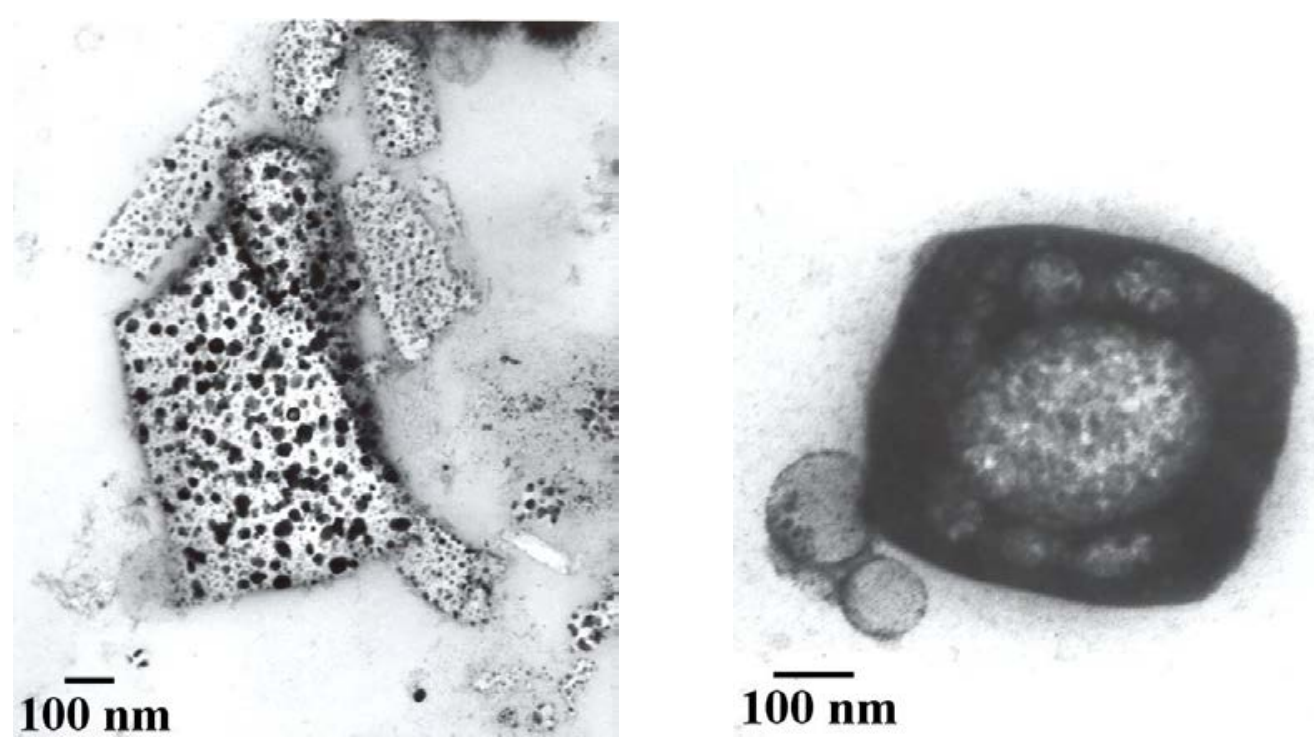

Fig.4. Cholesterol crystals preserving their sharp edges, albeit the decomposition of their inner layers into spherical particles (left) and a square-shaped cholesterol particle melting to form spherical droplets during TEM analysis (right). 

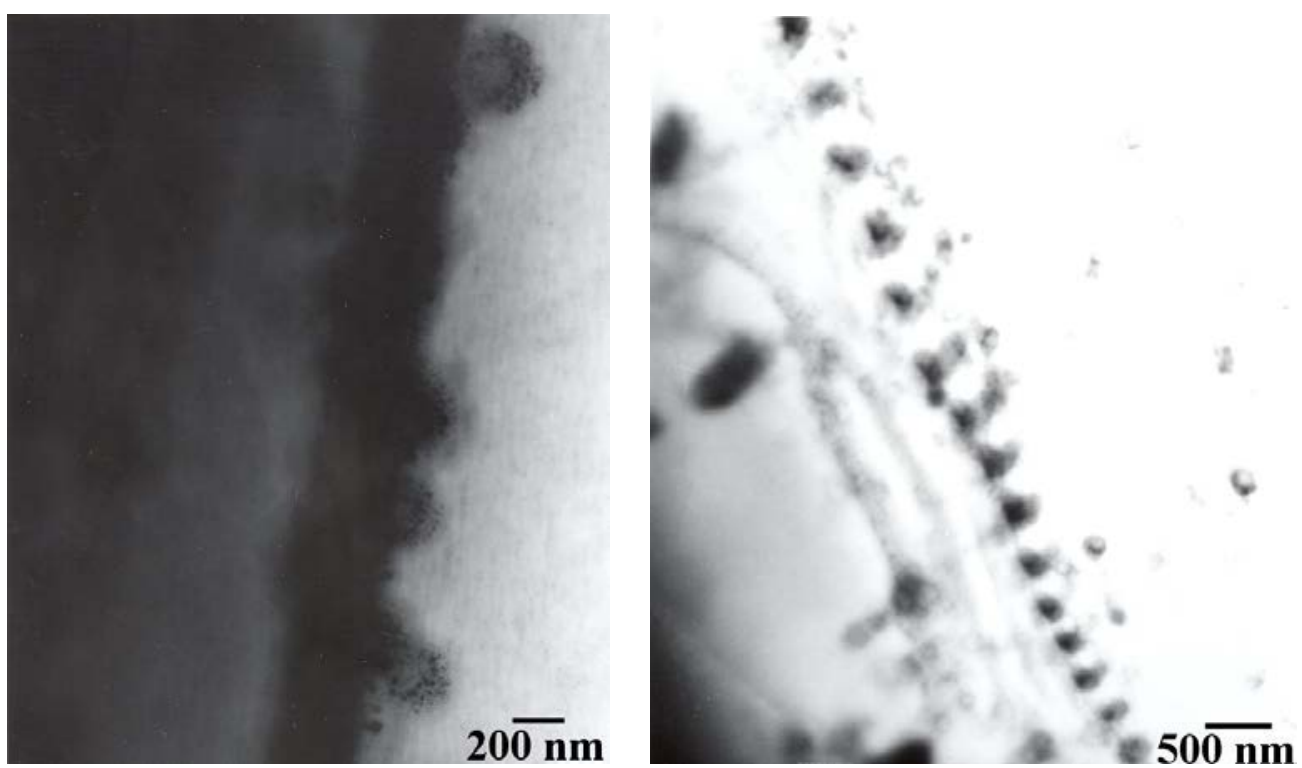

Fig.5. A needle-shaped cholesterol crystal transforming into an array of melted spherical droplets in the course of TEM analysis.

Crystallization of cholesterol within SDS- and CTAB-comprising 1hexanol/aqueous emulsions under specific conditions also results in the formation of well-dispersed and uniformly sized particles. Particles of unidentified composition, ranging $1-2 \mu \mathrm{m}$ in size, prepared in unstable, biphase-settling SDS/1-hexanol/water emulsion, according to the procedure presented in section 2.2, are shown in Fig.6. However, after prolonged aging of the still biphase mixture, up to $~ 4 \mathrm{~h}$, the initial particles, typical of their poriferous, spongy substructure and irregular but curved shapes, transform into larger, flower-like structures (with $\sim 10 \mu \mathrm{m}$ in radii), followed by the transition from whitish cholesterol curtain positioned at the alcohol-water interface on the bottom, aqueous phase side to white-colored upper, alcoholic phase (switching the medium transparency from alcoholic to aqueous phase, and its opacity in the opposite direction). SEM images of flower-shaped cholesterol crystals obtained by such aging of SDS-emulsified, hexanol/water biphase dispersion, consisting of platelet-shaped substructural units, are shown in Fig.7. Previously, many cases of cholesterol preparations, particularly within model bile environments and lecithin-containing emulsions, wherein transient micellar, vesicular and lamellar structures as metastable cholesterol carriers transform to plate-shaped cholesterol morphologies with time, were reported $^{27,28}$. The spongy globules obtained herein could similarly present either surfactant-comprising particles or an intermediate phase in the process of formation of larger aggregates composed of elongated cholesterol platelets. 


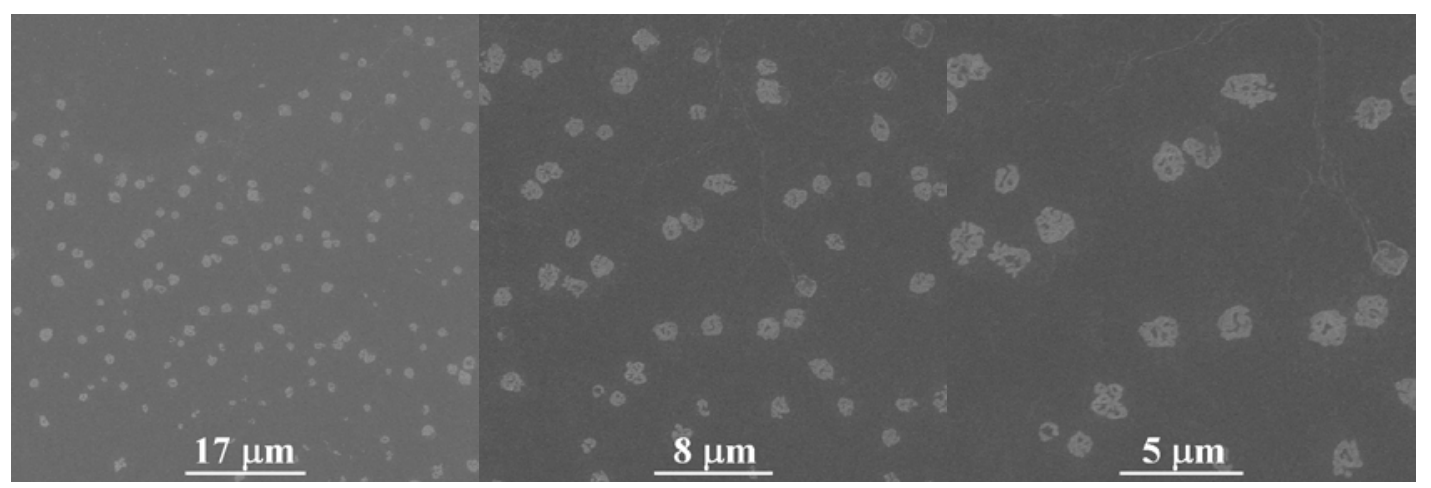

Fig. 6. Particles obtained by precipitation of cholesterol in SDS/1-hexanol/water emulsion.

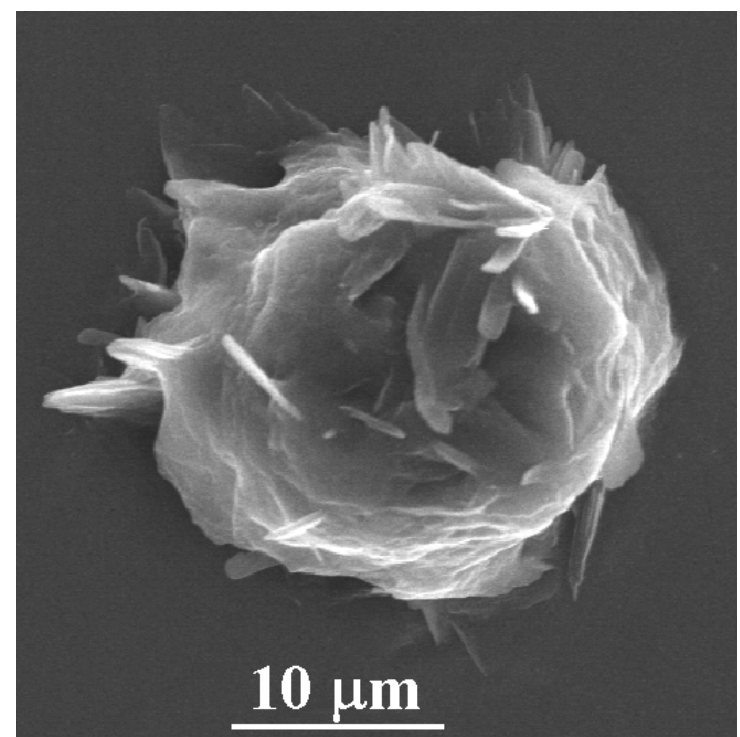

Fig.7. Individual flower-shaped cholesterol crystal obtained by 4h aging of non-agitated SDS-emulsified, hexanol/water biphase mixture.

SEM images of spherical cholesterol crystals, almost uniformly measuring $~ 10$ $\mu \mathrm{m}$ in diameter, prepared with using CTAB/1-hexanol/water emulsion as a parent precipitation medium, and evidently composed of layered, cake-like substructure, are shown in Figs.8-9. Comparing to the previous presented cases, nuclei growth rate is herein supposedly favored comparing to the rate of seed formation, resulting in the initial crystallization of millisized, plate-shaped cholesterol deposits that may only subsequently break into numerous spherical cholesterol islands. The mechanism of cleavage of cholesterol particles through the collisions of spherical emulsion droplets with cholesterol layers may be invoked in this case. Typical flat cholesterol deposits, being broken into numerous spherical islands by the influence of emulsion droplets, are presented in Fig.10. 


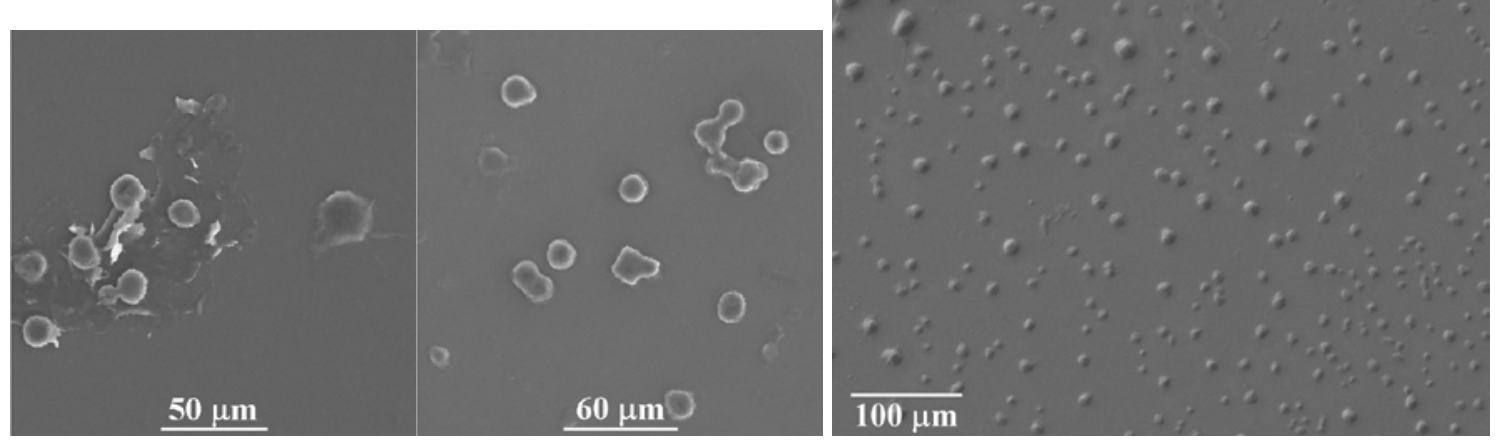

Fig.8. Round-shaped cholesterol crystals obtained by their precipitation in CTAB/1-hexanol/water emulsion.
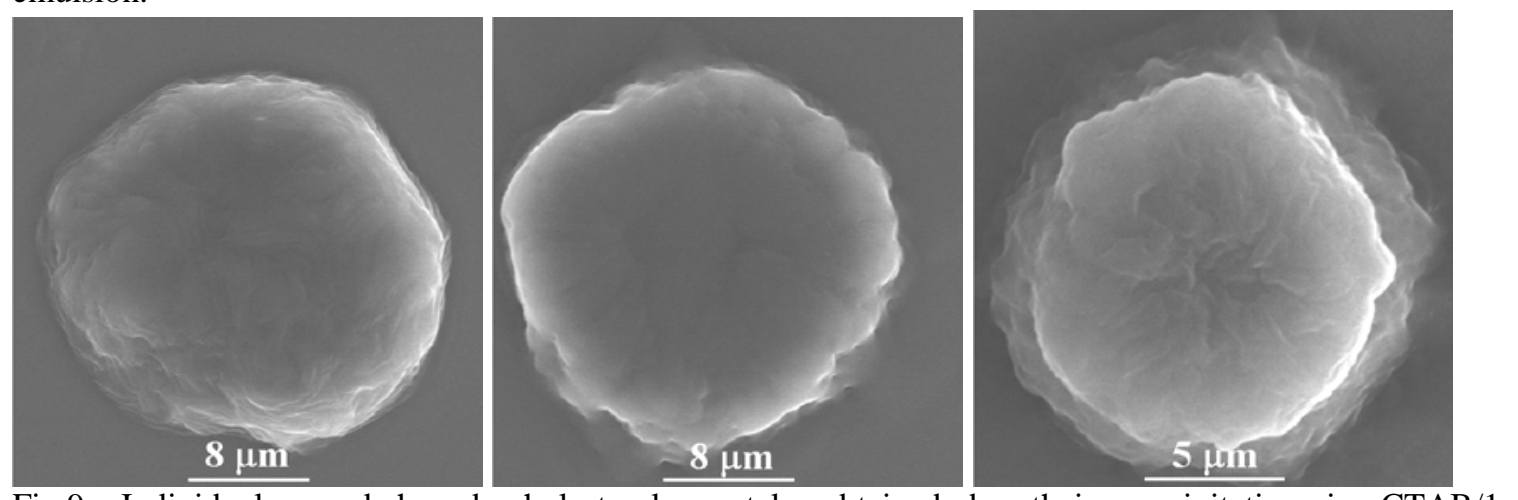

Fig.9. Individual round-shaped cholesterol crystals obtained by their precipitation in CTAB/1hexanol/water emulsion, evidently composed of layered, cake-like substructure.

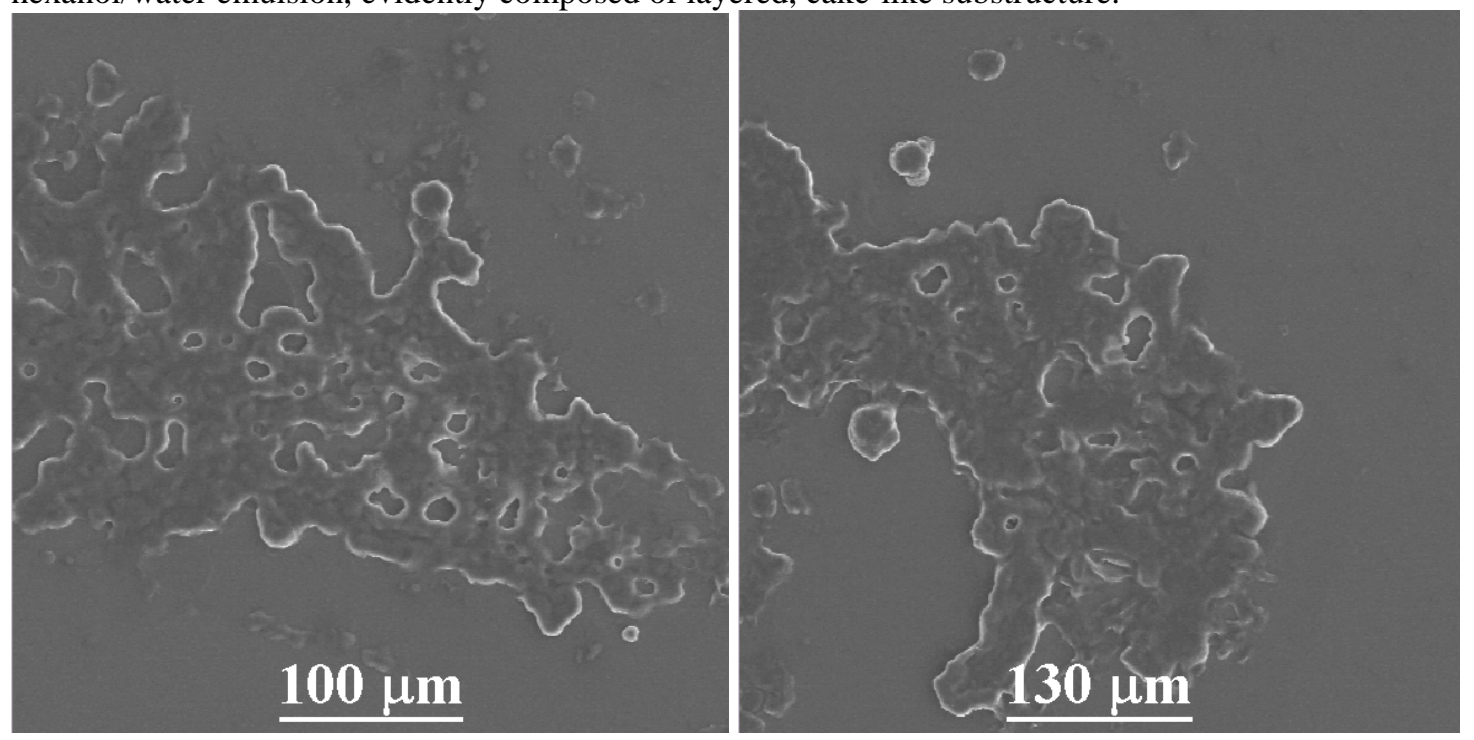

Fig.10. Cholesterol islands being chopped off from millisized initial cholesterol layers by the action of emulsion droplet dynamics.

The fourth method of precipitation presented in section 2.2, employing CTAB/1hexanol/water water-in-oil emulsion yields similar aggregates of spherical cholesterol "islands" with $\sim 50 \mu \mathrm{m}$ in size, as can be evidenced from Fig. 11. 


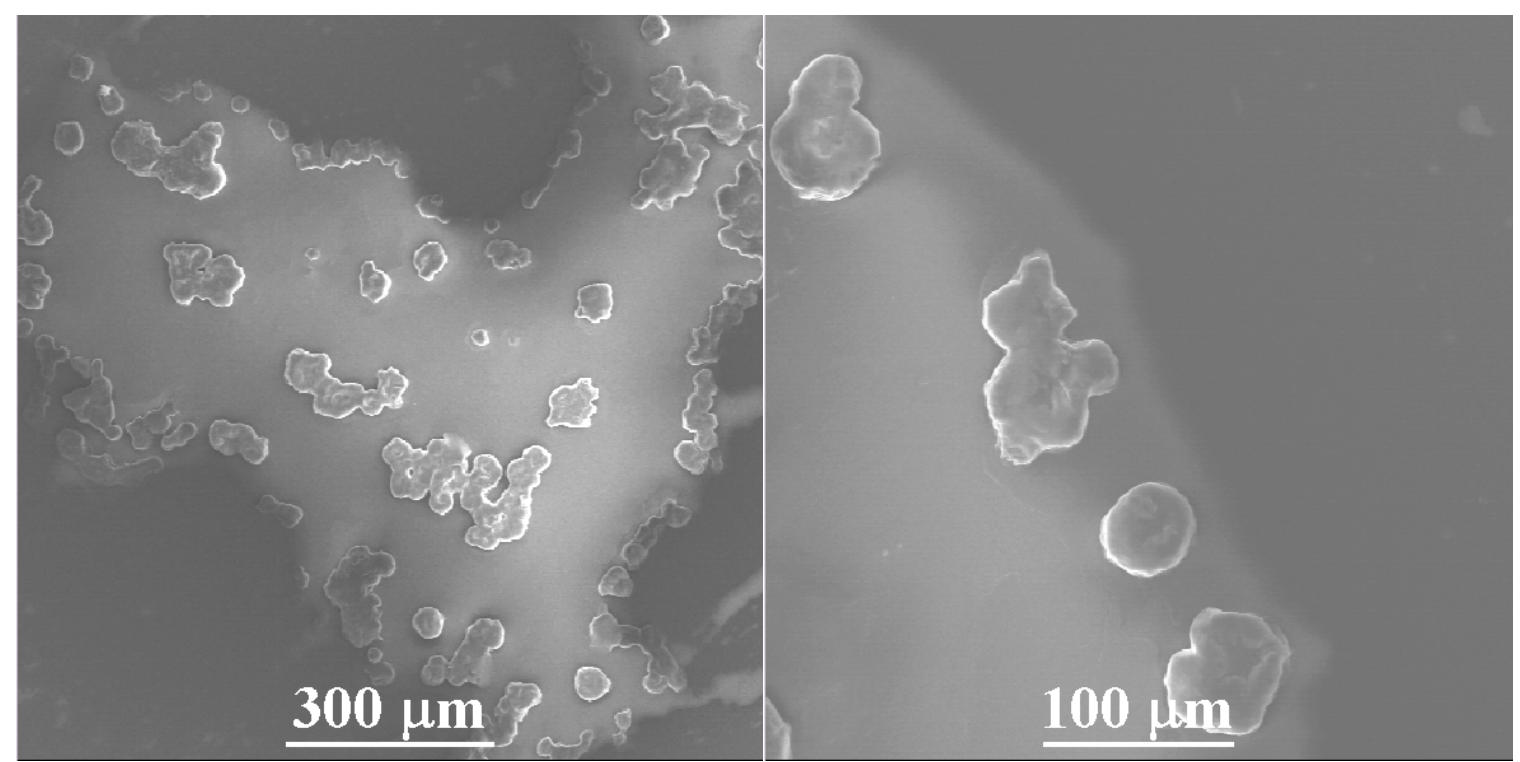

Fig.11. Microsized cholesterol spheroids prepared in accordance with the fourth method in section 2.2.

\section{Conclusion}

In accordance with the presented results, spatial restriction effects of micellar multi-molecular configurations during cholesterol crystallization can be considered as significant enough to either limit and direct growth of cholesterol crystals into circular micellar interior shapes or break initial or transient layered cholesterol precipitates into oval-shaped particles. All the investigated emulsion compositions under particular conditions offer possibilities for obtaining smaller and even submicron-sized cholesterol particles instead of large, millisized cholesterol crystalline deposits. Such an observation is consistent with the biological role of in vivo micelles and vesicles involved in the natural solubilization of gallbladder cholesteric deposits. Whereby previous investigations aimed at production of spherical cholesterol morphologies, based on the application of various solvents, surface active agents and synthesis approaches, in each given case resulted in plate-shaped cholesterol crystals and aggregates, it is shown hereby that cholesterol precipitation from dilute solutions may within specific chemical environments and compositions thereof indeed yield spherical cholesterol particles.

\section{References:}

1. F. R. Maxfield, I. Tabas - "Role of Cholesterol and Lipid Organization in Disease", Nature 438, $612-21$ (2005).

2. A. Elizabeth, C. Joseph, M. A. Ittyachen - »Growth and Micro-Topographical Studies of Gel Grown Cholesterol Crystals«, Bulletin of Materials Science 24 (4) 431 - 434 (2001).

3. N. Garti, L. Karpuj, S. Sarig - "Correlation between Crystal Habit and the Composition of Solvated and Nonsolvated Cholesterol Crystals”, Journal of Lipid Research 22, 785 - 791 (1981).

4. G. L. Flynn, Y. Shah, S. Prakongpan, K. H. Kwan, W. I. Higuchi, A. F. Hofmann - "Cholesterol Solubility in Organic Solvents”, Journal of Pharmaceutical Sciences 68 (9) 1090 - 1097 (1979).

5. R. J. Jandacek, M. R. Webb, F. H. Mattson - "Effect of an Aqueous Phase on the Solubility of Cholesterol in an Oil Phase”, Journal of Lipid Research 18, 203 - 210 (1977).

6. S. N. Kalkura, S. Devanarayanan - "Growth of Cholesterol Crystals in Silica Gel", Journal of Materials Science Letters 5, 741 - 741 (1986). 
7. P. A. Monzon, E. D. Siebert - "The Effect of Electrolytes on Cholesterol Crystal Growth in a Dynamic System”, Book of Abstracts, $215^{\text {th }}$ ACS National Meeting, Dallas, March 29 - April 2 (1998).

8. J. Ahumada, E. D. Siebert - "Cholesterol Crystal Growth from a Dynamic and Static Solvent System”, Book of Abstracts, $211^{\text {th }}$ ACS National Meeting, New Orleans, LA, March 24-28 (1996).

9. N. M. Sandarac, M. Ashok, N. Kalkura - »Observation of Cholesterol Nucleation in a Magnetic Field «, Acta Crystallographica D58, 1711 - 1714 (2002).

10. M. C. Frincu, S. D. Fleming, A. L. Rohl, J. A. Swift - »The Epitaxial Growth of Cholesterol Crystals from Bile Solutions on Calcite Substrates«, Journal of the American Chemical Society 126, 7915 - 7924 (2004).

11. M. C. Frincu, R. E. Sharpe, J. A. Swift - »Epitaxial Relationships between Cholesterol Crystals and Mineral Phases: Implication for Human Disease«, Crystal Growth \& Design 4 (2) 223 - 226 (2004).

12. B. M. Craven - "Crystal Structure of Cholesterol Monohydrate”, Nature 260, 727 - 9 (1976).

13. B. E. Cham - "Plaque Cholesterol and Calcium: the Value of EBCT in the Detection of Coronary Artherosclerosis”, European Journal of Clinical Investigation 31 (6), 467 - 468 (2001).

14. D. F. Laird, M. R. Mucalo, Y. Yokogawa - "Growth of Calcium Hydroxyapatite (Ca-HAp) on Cholesterol and Cholestanol Crystals from a Simulated Body Fluid: A Possible Insight into the Pathological Calcifications Associated with Artherosclerosis", Journal of Colloid and Interface Science 295, 348 - 363 (2006).

15. T. Nishioka, S. Tazuma, G. Yamashita,G. Kajiyama - "Quantitative Assessment of Comparative Potencies of Cholesterol-Crystal-Promoting Factors: Relation to Mechanistic Characterization”, Biochemical Journal 332, 343 - 350 (1998).

16. T. Nishioka, S. Tazuma, G. Yamashita, G. Kajiyama - "Partial Replacement of Bile Salts Causes Marked Changes of Cholesterol Crystallization in Supersaturated Model Bile Systems", Biochemical Journal 340, 445 - 451 (1999).

17. K. J. V. Erpecum, P. Portincasa, M. Gadella, B. J. M. V. De Heijning, G. P. V. B. Henegouwen, W. Renooij - "Effects of Bile Salt Hydrophobicity on Crystallization of Cholesterol in Model Bile”, European Journal of Clinical Investigation 26 (7) 602 (1996).

18. N. T. Saraswathi, F. D. Gnanam - "Effect of Medicinal Plants on the Crystallization of Cholesterol”, Journal of Crystal Growth 179, 611 - 617 (1997).

19. R. M. Epand, D. W. Hughes, B. G. Sayer, N. Borochov, D. Bach, E. Wachtel - "Novel Properties of Cholesterol-Dioleoylphosphatidylcholine Mixtures”, Biochimica et Biophysica Acta 1616, 196 -208 (2003).

20. J. Ahumada, E. D. Siebert - "Cholesterol Crystal Growth from a Dynamic and Static Solvent System”, Book of Abstracts, 213 ${ }^{\text {th }}$ ACS National Meeting, San Francisco, April 13-17 (1997).

21. L. Christiansen, M. Karjalainen, T. Seppanen-Laakso, R. Hiltunen, J. Yliruusi - "Effect of $\beta$ Sitosterol on Precipitation of Cholesterol from Non-Aqueous and Aqueous Solutions", International Journal of Pharmaceutics 254 (2) 155 - 166 (2003).

22. R. S. Abendan, J. A. Swift - "Surface Characterization of Cholesterol Monohydrate Single Crystals by Chemical Force Microscopy”, Langmuir 18, 4847 - 4853 (2002).

23. V. Uskoković, E. Matijević - "Uniform Particles of Pure and Silica Coated Cholesterol”, Journal of Colloid and Interface Science (accepted, in press, 2007).

24. C. W. Price - "The Electrophoretic Behaviour of Lecithin-Cholesterol Dispersions", Biochemical Journal 27 (6) 1789 - 1792 (1933).

25. C. R. Loomis, G. G. Shipley, D. M. Small - "The Phase Behavior of Hydrated Cholesterol”, Journal of Lipid Research 20, 525 - 535 (1979).

26. F. Debuigne, L. Jeunieau, M. Wiame, J. B. Nagy - "Synthesis of Organic Nanoparticles in Different W/O Microemulsions", Langmuir 16, 7605 - 7611 (2000).

27. D. L. Gantz, D. Q. H. Wang, M. C. Carey, D. M. Small - "Cryoelectron Microscopy of a Nucleating Model Bile in Vitreous Ice: Formation of Primordial Vesicles", Biophysical Journal 76 (3) 1436-1451 (1999).

28. F. M. Konikoff, D. E. Cohen, M. C. Carey - "Filamentous Crystallization of Cholesterol and its Dependence on Lecithin Species in Bile”, Molecular Crystals and Liquid Crystals Science and Technology, Section A: Molecular Crystals and Liquid Crystals 248, 799 - 804 (1994). 